\title{
A commentary on "Age-associated differences in macrophage response in a vaginal wound healing rat model"
}

\author{
Ghazaleh Rostaminia ${ }^{1}$
}

Received: 19 February 2020 / Accepted: 26 February 2020 / Published online: 11 March 2020

(C) The International Urogynecological Association 2020

This basic science animal study [1] was aimed at investigating the effect of age on inflammatory processes, specifically the macrophage response, involved in vaginal wound healing. A total of 25 young virgin female Fischer rats ( 12 weeks old) and 25 old rats (12 months), underwent a standardized 9-mm posterior midline vaginal incision. Tissue samples were taken for histological analysis on days 1, 3, 7, 14, and 30 post-injury. At each time point, 5 rats were included in each experimental group. The parameters evaluated included the wound area, macrophage number, and expression of inflammatory markers, including tumor necrosis factor alpha (TNF $\alpha)$, inducible nitric oxide synthase (iNOS), CCR7/CD197, arginase I, and CD163/M130.

Microscopic examination of the vaginal wounds over time demonstrated a clear difference between young and old rats in spontaneous healing capacity. The average wound area in young rats 1 day after injury was significantly smaller than in the old rats $\left(16.5 \pm 1.7\right.$ vs $\left.23.8 \pm 1.5 \mathrm{~mm}^{2}, P<0.05\right)$. Three days post-injury, wounds were sealed in the young rats, but remained open $\left(13.5 \pm 1.5 \mathrm{~mm}^{2}\right)$ in the old rats. By day 14 post-injury, wounds were closed in the old rats as well. The old rats had a greater macrophage response to injury than the young rats. The number of $\mathrm{CD} 68^{+}$macrophages was significantly higher between days 3 and 30 post-injury. Furthermore, the rate of decrease in macrophage infiltration was faster in the young compared with the old rats.
In summary, this study observed differences in the inflammatory response during the wound-healing process in the vagina of old and young rats. In a prior study looking at biomechanical properties [2], it was shown that age was associated with an impaired wound healing response as well, resulting in decreased tissue strength 30 days post-injury. This outcome coincides with the prolonged inflammatory response and expression of macrophage migration inhibitory growth factor (MIF) that was highlighted in the current study. Although the growing multidisciplinary field of tissue engineering is aimed at predictably regenerating, enhancing, or replacing damaged tissues for a variety of conditions caused by trauma, disease, and old age, this finding can help with the development of targeted, effective treatments to achieve stronger tissues and improved surgical outcomes.

\section{References}

1. Ben Menachem-Zidon O, Parkes I, Chill HH, et al. Age-associated differences in macrophage response in a vaginal wound healing rat model. Int Urogynecol J. 2020. https://doi.org/10.1007/s00192-02004266-9.

2. Shveiky D, Iglesia CB, Sarkar Das S, Menachem-Zidon OB, Chill $\mathrm{HH}$, Ji H, Sandberg K. Age-associated impairments in tissue strength and immune response in a rat vaginal injury model. Int Urogynecol J. 2019. https://doi.org/10.1007/s00192-019-04008-6.

Ghazaleh Rostaminia

ghazalerostaminia@yahoo.com

1 North Shore University Health System, Skokie, IL, USA 